\title{
Proceedings of the EACL 2009 Workshop on Computational Linguistic Aspects of \\ Grammatical Inference
}

\section{CLAGI 2009}

\author{
30 March - 2009 \\ Megaron Athens International Conference Centre \\ Athens, Greece
}


Production and Manufacturing by

TEHNOGRAFIA DIGITAL PRESS

7 Ektoros Street

15235 Vrilissia

Athens, Greece

\section{PASCAL2}

(C)2009 The Association for Computational Linguistics

Order copies of this and other ACL proceedings from:

Association for Computational Linguistics (ACL)

209 N. Eighth Street

Stroudsburg, PA 18360

USA

Tel: +1-570-476-8006

Fax: +1-570-476-0860

acleaclweb.org 


\section{Preface}

We are delighted to present you with this volume containing the papers accepted for presentation at the $1^{\text {st }}$ workshop CLAGI 2009, held in Athens, Greece, on March 30 2009.

We want to acknowledge the help of the PASCAL 2 network of excellence.

Thanks also to Damir Ćavar for giving an invited talk and to the programme committee for the reviewing and advising.

We are indebted to the general chair of EACL 2009, Alex Lascarides, to the publication chairs, Kemal Oflazer and David Schlangen, and to the Workshop chairs Stephen Clark and Miriam Butt, for all their help. We are also grateful for Thierry Murgue's assistance when putting together the proceedings.

Wishing you a very enjoyable time at CLAGI 2009!

Menno van Zaanen and Colin de la Higuera

Clagi 2009 Programme Chairs 



\section{CLAGI 2009 Program Committee}

\section{Program Chairs:}

Menno van Zaanen, Tilburg University (The Netherlands)

Colin de la Higuera, University of Saint-Étienne, (France)

\section{Program Committee Members:}

Pieter Adriaans, University of Amsterdam (The Netherlands)

Srinivas Bangalore, AT\&T Labs-Research (USA)

Leonor Becerra-Bonache, Yale University (USA)

Rens Bod, University of Amsterdam (The Netherlands)

Antal van den Bosch, Tilburg University (The Netherlands)

Alexander Clark, Royal Halloway, University of London (UK)

Walter Daelemans, University of Antwerp (Belgium)

Shimon Edelman, Cornell University (USA)

Jeroen Geertzen, University of Cambridge (UK)

Jeffrey Heinz, University of Delaware (USA)

Colin de la Higuera, University of Saint-Étienne (France)

Alfons Juan, Polytechnic University of Valencia (Spain)

Frantisek Mraz, Charles University (Czech Republic)

Georgios Petasis, National Centre for Scientific Research (NCSR) "Demokritos" (Greece)

Khalil Sima' an, University of Amsterdam (The Netherlands)

Richard Sproat, University of Illinois at Urbana-Champaign (USA)

Menno van Zaanen, Tilburg University (The Netherlands)

Willem Zuidema, University of Amsterdam (The Netherlands) 



\section{Table of Contents}

Grammatical Inference and Computational Linguistics

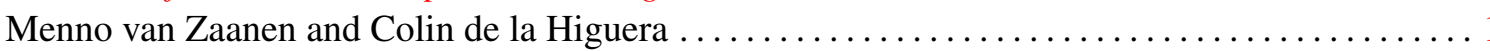

On Bootstrapping of Linguistic Features for Bootstrapping Grammars

Damir Ćavar . . . . . . . . . . . . . . . . . . . . . . . . . . . . . . . . . . . . . . . . . 5

Dialogue Act Prediction Using Stochastic Context-Free Grammar Induction

Jeroen Geertzen .............................................. 7

Experiments Using OSTIA for a Language Production Task

Dana Angluin and Leonor Becerra-Bonache ................................ 16

GREAT: A Finite-State Machine Translation Toolkit Implementing a Grammatical Inference Approach for Transducer Inference (GIATI)

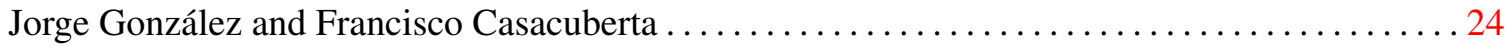

A Note on Contextual Binary Feature Grammars

Alexander Clark, Remi Eyraud and Amaury Habrard ............................ 33

Language Models for Contextual Error Detection and Correction

Herman Stehouwer and Menno van Zaanen ................................ 41

On Statistical Parsing of French with Supervised and Semi-Supervised Strategies

Marie Candito, Benoit Crabbé and Djamé Seddah................................. 49

Upper Bounds for Unsupervised Parsing with Unambiguous Non-Terminally Separated Grammars

Franco M. Luque and Gabriel Infante-Lopez...................................... 58

Comparing Learners for Boolean partitions: Implications for Morphological Paradigms

Katya Pertsova ................................................... 66 
